\title{
Claves Psicosociales para la Permanencia de la Víctima en una Relación de Maltrato
}

\section{Psychosocial Keys for the Permanence of the Victim in an Abusive Relationship}

\author{
Pedro J. Amor \\ Universidad Nacional de Educación a Distancia
}

\author{
Enrique Echeburúa \\ Universidad del País Vasco-CIBERSAM
}

Resumen. En este artículo se analizan los factores que están más vinculados a la permanencia de las víctimas en convivencia con el agresor (características de las víctimas y de la pareja agresora, junto con otras variables contextuales). Por otra parte, se describen brevemente las principales teorías explicativas referidas a este fenómeno: teorías centradas en el proceso de toma de decisiones, teorías referidas a la dependencia emocional de las víctimas, y teorías basadas en el estado emocional y las repercusiones psicopatológicas del maltrato. Finalmente, se alude a la importancia de respetar las decisiones de las víctimas con objeto de prevenir la victimización secundaria.

Palabras clave: violencia de pareja, mujeres maltratadas, permanencia en la relación de maltratado, toma de decisiones.

\begin{abstract}
This article deals with the factors that are most closely related to the permanence of battered women with batterer men (characteristics of victims and aggressors, along with other contextual variables). In addition, the main explanatory theories concerning this topic are briefly summarized: theories focusing on the process of decision making, theories concerning the victim's emotional dependency, and theories based on the emotional and psychopathological impact of abuse. Finally, we refer to the importance of respecting the decisions of the victims in order to prevent secondary victimization.

Key words: intimate partner violence, battered women, remaining in abusive relationships, decision-making.
\end{abstract}

\section{Introducción}

Un tema que ha despertado un gran interés en las últimas décadas es conocer las razones por las que una víctima de maltrato permanece con el agresor o, tras romper la relación, reanuda la convivencia con su ex pareja agresora (Aguirre, 1985; Anderson, 2003). Según Rhatigan, Street y Axsom (2006), muchas víctimas necesitan realizar múltiples intentos e implicarse en un proceso de toma de decisiones complejo para salir definitivamente de una relación violenta.

El conocimiento de las causas que influyen en las decisiones de las mujeres para permanecer con la pareja abusiva o para abandonar la relación contribuye a prevenir las agresiones graves y a reducir los síntomas psi-

La correspondencia sobre este artículo puede dirigirse a Enrique Echeburúa. Facultad de Psicología. Universidad del País Vasco. Avda. de Tolosa, 70.20018 San Sebastián (España). E-mail: enrique.echeburua@ehu.es. Teléfono: 943018329. 
copatológicos de la víctima, así como a fortalecerla cognitiva y emocionalmente. De esta forma, se pueden diseñar programas de intervención más efectivos (Rhatigan et al., 2006).

En este artículo se analizan brevemente los factores que están más vinculados a la permanencia de las víctimas con el agresor (características de las víctimas, de los agresores y del contexto de la relación), así como las principales teorías explicativas, que están centradas en el proceso de toma de decisiones, en la dependencia emocional de la víctima respecto al agresor y en las repercusiones psicológicas del maltrato crónico. Un fenómeno menos estudiado, y al que también se presta atención en este trabajo, es el referido a la victimización secundaria de las víctimas que continúan conviviendo o vuelven a convivir con el agresor.

\section{Factores vinculados a la permanencia}

En los últimos años se han realizado múltiples investigaciones para conocer los distintos factores relacionados con la permanencia de las víctimas en una relación de pareja violenta.

\section{a) Características de las víctimas}

Al margen de que cada mujer maltratada responde a una personalidad única y a un conjunto de circunstancias específicas, hay ciertos factores genéricos habitualmente presentes en gran parte de las víctimas (Rhatigan et al., 2006; Rhodes y Baranoff, 1998): a) creencias y actitudes tradicionales en cuanto al rol de la mujer y al compromiso moral que supone el matrimonio (por ejemplo, "el matrimonio es para siempre", "enfermo como está, no le puedo abandonar", etc.); b) baja autoestima; c) locus de control externo; d) presencia de apego ansioso; e) tendencia a culparse por la violencia sufrida o a exculpar al agresor de los episodios violentos; y f) resistencia a usar los servicios comunitarios disponibles (casas de acogida, servicios de apoyo, etc.).

Se han estudiado también otros factores (como haber sufrido abusos en la infancia, carecer de apoyo social o tener un nivel educativo y socioeconómico bajo), pero los resultados no son concluyentes (Rhatigan et al., 2006).

\section{b) Perfil de los agresores}

Aunque sea la mujer quien decida permanecer con el agresor o abandonarlo, las conductas y características de los agresores tienen una gran influencia en su proceso de toma de decisiones. Por otro lado, existen distintos tipos de agresores (Amor, Echeburúa y Loinaz, 2009; Cavanaugh y Gelles, 2005), que maltratan con diferente frecuencia e intensidad y que representan un mayor o menor riesgo para la integridad física y psicológica de las víctimas.

Desde esta perspectiva, las víctimas afectadas por niveles relativamente bajos o moderados de violencia aguantan más la convivencia con el agresor, tienden a buscar una justificación del maltrato del que son objeto o tratan de restablecer una relación de pareja armoniosa. En cambio, aquellas víctimas que sufren elevados niveles de violencia, o que observan en el agresor comportamientos indicativos de violencia grave (por ejemplo, humillar y atacar a la víctima o amenazarla de muerte, etc.), son las que más desean abandonar la relación (Rhatigan et al., 2006) pero, al mismo tiempo, las que más temen hacerlo por los diferentes riesgos que supone tal decisión (represalias, intentos de homicidio, etc.).

Otras circunstancias de los agresores que hacen más probable la permanencia de las víctimas son las siguientes: tener empleo y ser la principal fuente de ingresos económicos para la familia, acudir a tratamiento psicológico (Gondolf, 1988), estar comprometido con el cambio, no tener problemas con el alcohol y no presentar rasgos patológicos de personalidad (Stroshine y Robinson, 2003). 


\section{c) Relación de pareja y otras variables contextuales}

Uno de los factores más importantes es la interacción entre la pareja. La permanencia de la víctima en una relación de pareja violenta puede estar basada en determinadas pautas de interacción, tales como el ciclo de la violencia (Walker, 1979), las contingencias de reforzamiento en función de un patrón cíclico de interacción consolidado temporalmente (Long y McNamara, 1989) y la intermitencia entre el buen y el mal trato (Dutton y Painter, 1993). Este tipo de interacciones o de circunstancias fomentaría un vínculo emocional que supondría una gran barrera para el abandono de la relación.

Otras circunstancias tienen que ver con factores más psicosociales, tales como la dependencia económica del agresor (Aguirre, 1985), las dificultades laborales (Anderson, 2003), la presencia de hijos pequeños a cargo de la víctima, el aislamiento social y familiar, el desconocimiento de los recursos comunitarios existentes y la ausencia de alternativas reales en cuanto al empleo.

Por otro lado, es más probable que las víctimas tengan más deseos de abandonar al agresor ante las siguientes circunstancias: a) insatisfacción con su relación de pareja y menor compromiso con la relación (Rhatigan et al., 2006); b) intentos fallidos para resolver conflictos (Bauserman y Arias, 1992); c) menor número de restricciones en el caso de separarse, tanto objetivas (por ejemplo, ausencia de hijos y de propiedades compartidas) como subjetivas (por ejemplo, apoyo social y disponibilidad de alternativas) (Gordon, Burton y Porter, 2004); d) disponibilidad de más recursos económicos (Aguirre, 1985, Lesser, 1990); y e) padecimiento de la violencia con más intensidad/frecuencia y desde hace tiempo (Holtzworth-Munroe, Smultzer, Bates y Sandin, 1997).

\section{Teorías explicativas de la permanencia}

Existen múltiples teorías que tratan de explicar la permanencia de una mujer en una relación violenta, así como la probabilidad de abandonar la convivencia con el agresor. Se comentan a continuación los tres principales grupos de teorías, que ponen el acento en unas u otras variables (Figura 1).

Figura 1. Principales teorías explicativas sobre la permanencia de la víctima en convivencia con el agresor o sobre el abandono de la relación de pareja

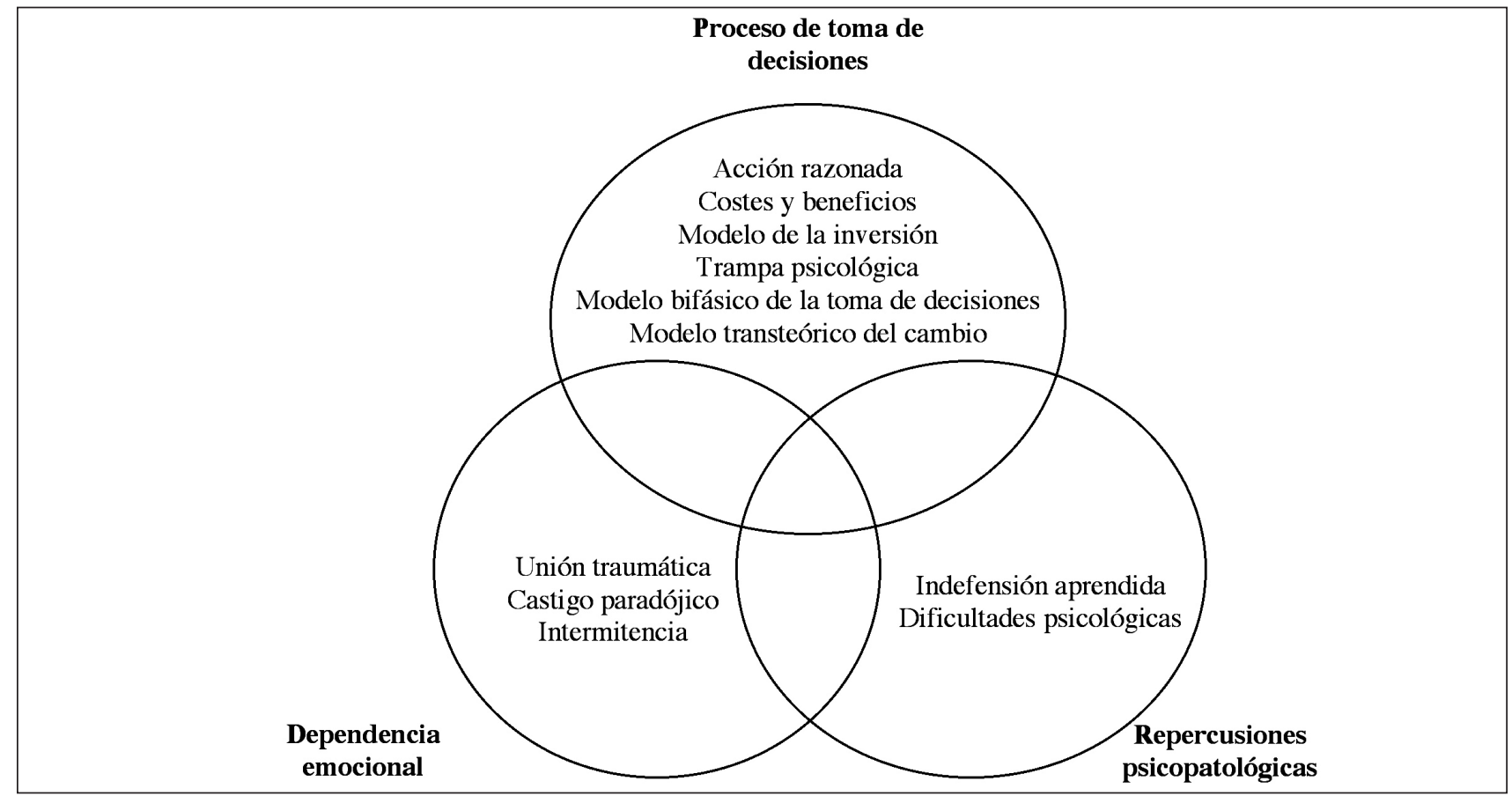




\section{a) El proceso de toma de decisiones}

Estas teorías toman en consideración diversos aspectos: el balance que hacen las víctimas sobre los costes y beneficios de proseguir o de abandonar la relación, las redes de apoyo social y las alternativas disponibles si deciden abandonar la relación, el grado de satisfacción con su relación de pareja y las expectativas de lograr una convivencia armoniosa a través de la inversión de más esfuerzos y tiempo. También cuentan el grado de compromiso y de motivación, así como la convicción de cada víctima para permanecer o abandonar la relación de pareja.

- Teoría de la acción razonada/conducta planificada (Ajzen, 1985; Fishbein y Ajzen, 1975; Pfouts, 1978): la mujer decide abandonar o continuar con su relación de pareja (intención conductual) en función de la norma social (redes de influencia social que pueden condicionar su decisión) y de las expectativas de los resultados, es decir, de la valoración de los costes (desventajas) y beneficios (ventajas) percibidos en función de las posibles alternativas que se le presentan. Así, sería más probable que una víctima de maltrato decidiera seguir en convivencia con su pareja si ante el abandono teme sufrir más violencia, padecer problemas económicos o quedarse sin la custodia de los hijos.

- Modelo de inversión (Rusbult, 1980, 1983; Rhatigan y Axsom, 2006): las víctimas que se sienten más comprometidas dentro de la relación de pareja tienen una mayor probabilidad de permanecer con ella. El nivel de compromiso de la víctima se mide a partir de tres factores: grado de satisfacción, alternativas disponibles e inversión realizada (por ejemplo, hijos en común, tiempo de convivencia, estabilidad económica, etc.). El grado de compromiso estaría representado mediante la siguiente ecuación:

\section{Compromiso $=$ Satisfacción - Alternativas + Inversión}

- Trampa psicológica (Brockner y Rubin, 1985; Strube, 1988): la mujer maltratada tiene la esperanza de que cese el maltrato y cree que, invirtiendo más esfuerzos y tiempo, puede restablecer una relación de pareja armoniosa. Según esta teoría, al inicio del maltrato la víctima invierte muchos esfuerzos para que cese esta pauta de conducta, pero más adelante, cuando los episodios aumentan en frecuencia e intensidad, la mujer puede plantearse abandonar la relación. Sin embargo, muchas de ellas creen que hay posibilidades de que la relación mejore y, por ello, pueden invertir aún más esfuerzos para lograr su objetivo. Asimismo, cuantos más esfuerzos y tiempo inviertan (y hayan invertido en el pasado) para lograr una relación armoniosa, menor es la probabilidad de que se produzca el abandono de la relación de pareja.

- Modelo bifásico de la toma de decisiones (Choice y Lamke, 1997): las víctimas deciden continuar o concluir la relación en función de la respuesta a dos preguntas clave: 1) ¿estaré mejor fuera de la relación?; y 2) ¿seré capaz de salir de ella con éxito? (Figura 2). La contestación a la primera pregunta está modulada por cuatro factores: a) el grado de satisfacción con la relación de pareja; b) la inversión realizada; c) la calidad de las alternativas disponibles; y d) la presión ambiental y familiar para continuar o concluir la relación. La respuesta a la segunda cuestión va a depender de los recursos psicológicos disponibles y de los apoyos comunitarios a nivel socioeconómico y jurídico. Si la mujer maltratada responde negativamente a cualquiera de las dos preguntas, tenderá a continuar dentro de la relación de pareja.

- Modelo transteórico del cambio (Prochaska y DiClemente, 1982): adaptado por Brown (1997) al ámbito del maltrato, se centra en el análisis de las etapas del cambio por las que pasa una víctima para abandonar una relación de pareja violenta. Las diferentes etapas son las siguientes: precontemplación (la víctima tiende a minimizar o negar el problema y sus consecuencias); contemplación (hay una gran ambivalencia y disonancia cognitiva entre el afecto y el maltrato que sufre); preparación (disminuye la ambivalencia, se tiene más conciencia del problema y aumentan los deseos de cambio); acción (se realizan cambios, se toman decisiones, se emprenden acciones legales, etc.), mantenimiento y terminación. Las características de cada etapa pueden ayudar a comprender mejor las circunstancias en las que se encuentra cada víctima y las decisiones que suelen tomar en cada momento, así como a diseñar las estrategias de intervención más adecuadas. 
Figura 2. Modelo conceptual del proceso de toma de decisiones en mujeres maltratadas (Choice y Lamke, 1997)

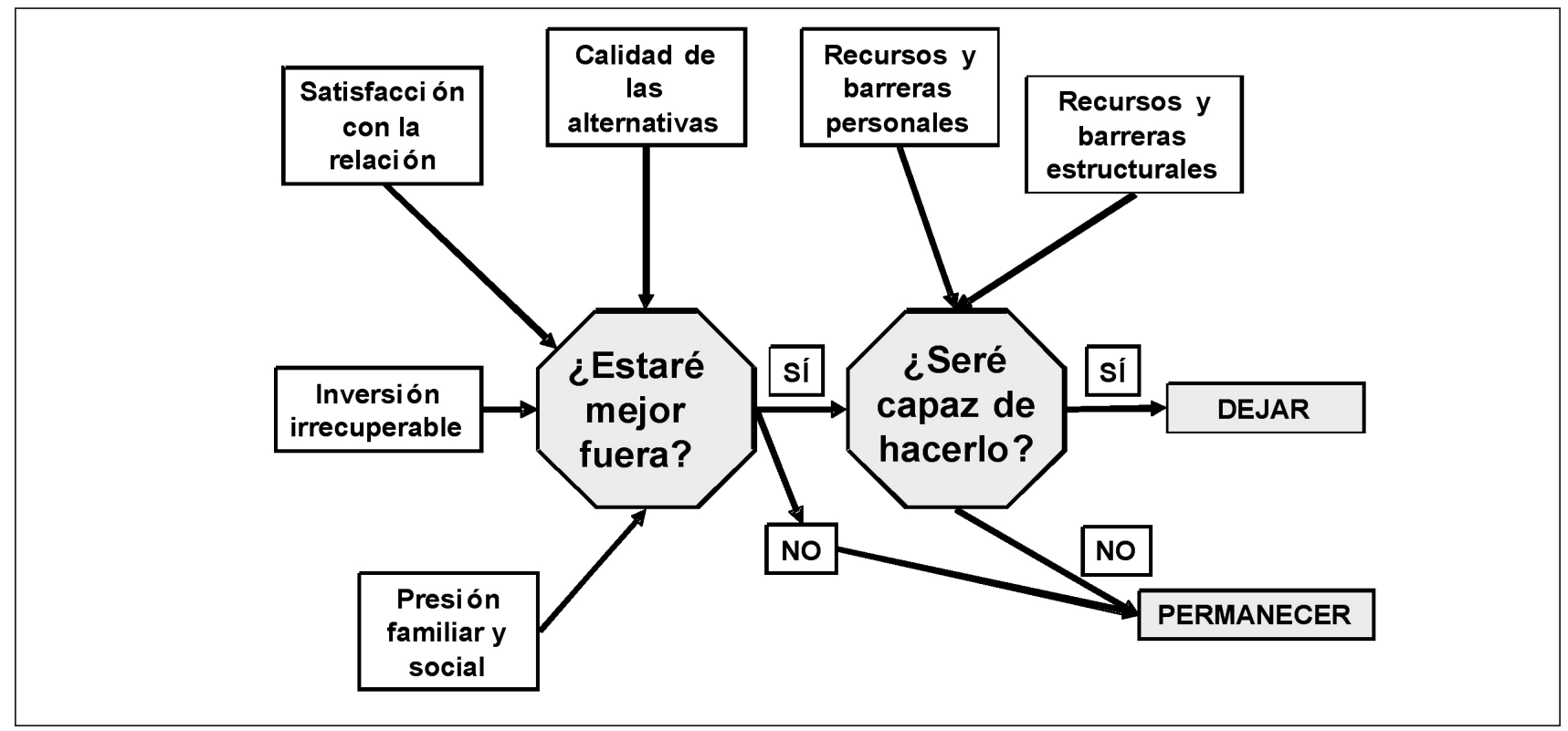

\section{b) La dependencia emocional}

En cierto modo, las víctimas de maltrato permanecen en convivencia con su pareja por el vínculo emocional establecido, que, entre otros aspectos, está basado en la intermitencia entre el buen y el mal trato ejercido por el agresor.

- Teoría de la unión traumática (Dutton y Painter, 1981) y modelo de intermitencia (Dutton y Painter, 1993): enfatizan la asimetría de poder entre el agresor y la víctima (mujer). El fuerte apego establecido entre la víctima y el agresor se debe al carácter extremo del buen y el mal trato, así como a la yuxtaposición temporal de ambos extremos. Además, cuanto más maltrato sufre la mujer, más se resiente su autoestima, y quizás tenga una mayor necesidad de su pareja, convirtiéndose finalmente en interdependencia.

- Modelo del castigo paradójico (Long y McNamara, 1989): va más allá de la teoría del ciclo de la violencia de Walker $(1979,1984)$ y sostiene que la mujer permanece en la situación de maltrato debido a las contingencias de reforzamiento que se establecen en función de un patrón cíclico de interacción que está consolidado en el tiempo y que consta de cinco fases: creación de la tensión, descarga de la tensión por parte del maltratador, escape de la víctima, arrepentimiento del agresor y, finalmente, "reconciliación".

\section{c) Las repercusiones psicopatológicas del maltrato}

- Teoría de la indefensión aprendida (Seligman, 1975; Walker, 1979, 1984): la persona que sufre maltrato de forma impredecible e incontrolable durante un periodo prolongado de tiempo llega a un estado tal de indefensión y de déficit en diferentes áreas (motivación, cognición y afecto) que hace más probable su permanencia dentro de esa relación. Asimismo su malestar interfiere gravemente en su proceso de toma de decisiones.

- Modelos psicológico y ambiental de la permanencia de la víctima (Foa, Cascardi, Zoellner y Feeny, 2000): la carencia de estrategias de afrontamiento adecuadas en la víctima (salud, autoestima, estilo optimista, etc.), las repercusiones psicopatológicas experimentadas por el maltrato crónico (TEPT, síntomas ansiosodepresivos, etc.) y los factores sociales (falta de empleo, precariedad económica, etc.) interactúan con las res- 
puestas específicas de la víctima en el ámbito de la relación (dependencia emocional, expectativas negativas de cambio, insatisfacción con la relación, etc.).

\section{Las decisiones de las víctimas y la victimización secundaria}

Las decisiones de las mujeres maltratadas -por ejemplo, separarse del agresor, seguir conviviendo con él o abandonarlo y a los pocos meses reanudar la relación-, no deben ser objeto de victimización secundaria, y menos aún por los profesionales que tienen contacto con ellas desde diferentes ámbitos.

Más que juzgar la decisión tomada por la mujer, conviene tener una visión más amplia y comprensiva, tomando en consideración las circunstancias de las víctimas (riesgos, necesidades, temores, otras vivencias emocionales, fase del cambio en la que se encuentran, etc.).

De hecho, un componente básico del fortalecimiento de la víctima es que ella sea capaz de tomar sus propias decisiones. Salir de una relación violenta es un proceso de cambio dinámico que, en determinados casos, requiere múltiples intentos antes de conseguirlo definitivamente (Goodman, Dutton, Weinfurt y Cook, 2003). Las víctimas pueden inicialmente minimizar la violencia, culparse por el deterioro de la relación, atribuir erróneamente la violencia a una situación pasajera y mostrar una ambivalencia (deseos de abandonar a quien les agrede y, al mismo tiempo, de seguir conviviendo con la misma persona) (Brown, 1997).

Conocer estos aspectos puede contribuir a la prevención de la victimización secundaria, así como ayudar en la selección de las estrategias de intervención más adecuadas en cada momento. Por ejemplo, puede ser de gran ayuda, en las primeras fases del cambio, fortalecer los recursos psicológicos de las víctimas (autoestima, asertividad, habilidades sociales, toma de decisiones, etc.) y facilitarles una mayor satisfacción vital como primer paso del proceso (Ben-Porat e Itzhaky, 2008).

Según Bell, Goodman y Dutton (2007), el abandono de la relación, sin la existencia de un apoyo social o comunitario, puede que no sea la mejor elección para todas las víctimas. Algunas mujeres separadas pueden seguir sufriendo maltrato (acoso, amenazas, agresiones, etc.) o tenerse que enfrentar a otros estresores (buscar trabajo, luchar por la custodia de los hijos, verse obligadas a cambiar de entorno y de actividades, etc.).

\section{Conclusiones}

El abandono de una relación de pareja violenta supone una decisión extremadamente compleja y difícil de tomar. En cada caso existen unas circunstancias vinculadas a la víctima (por ejemplo, creencias y actitudes tradicionales, sentimientos de baja autoestima y de culpa, malestar emocional, miedo extremo, etc.), al agresor (por ejemplo, presencia de características antisociales, gravedad de la violencia, "arrepentimientos" intermitentes, amenazas de suicidio o de muerte a su mujer e hijos, etc.) y a la relación (dependencia económica, aislamiento, presiones familiares o sociales, dificultades para acceder a recursos comunitarios, etc.). Este conjunto de circunstancias, que tienen un peso diferente de unas personas a otras, dificultan enormemente el abandono de la relación (Echeburúa, Amor y Corral, 2002; Rhodes y Baranoff, 1998; Salber y Taliaferro, 2000).

En este sentido, la decisión de permanecer conviviendo con el agresor o de abandonar la relación supone un proceso dinámico (Goodman et al., 2003) que está condicionado por múltiples factores socioeconómicos, cognitivos, emocionales y psicopatológicos (Rhatigan et al., 2006). Conviene, por ello, a los profesionales (jueces, psicólogos, trabajadores sociales, educadores o policías) tener una visión más comprensiva e idiográfica acerca de las decisiones que toma cada víctima según el momento y sus circunstancias.

Por último, se plantean algunos retos de futuro. Así, por ejemplo, conviene analizar con detalle el curso longitudinal de los síntomas físicos y psicopatológicos en las mujeres maltratadas que conviven con su pareja agre- 
sora en comparación con aquellas que abandonan temporal o definitivamente la relación (Amor, Bohórquez y Echeburúa, 2006). También es una tarea necesaria estudiar a mujeres maltratadas pertenecientes a diferentes ámbitos culturales (por ejemplo, a víctimas inmigrantes), determinar las dificultades específicas que tienen para salir de la relación de pareja y precisar estrategias concretas de ayuda en este contexto (Gharaibeh y Oweis, 2009).

\section{Referencias}

Aguirre, B.E. (1985). Why do they return? Abused wives in shelters. Social Work, 30, 350-354.

Ajzen, I. (1985). From intentions to actions: A theory of planned behavior. En J. Kuhl y J. Beckman (Eds.), Action control: From cognition to behaviour (pp. 11-39). Heidelberg, Federal Republic of Germany: Springer.

Amor, P.J., Bohórquez, I. y Echeburúa, E. (2006). ¿Por qué y a qué coste físico y psicológico permanece la mujer junto a su pareja maltratadora. Acción Psicológica, 4, 129-154.

Amor, P.J., Echeburúa, E. y Loinaz, I. (2009). ¿Se puede establecer una clasificación tipológica de hombres violentos contra su pareja? International Journal of Clinical and Health Psychology, 9, 519-539.

Anderson, D.J. (2003). The impact on subsequent violence of returning to an abusive partner. Journal of Comparative Family Studies, 34, 93-112.

Bauserman, S.K. y Arias, A. (1992). Relationships among marital investment, marital satisfaction, and marital commitment in domestically victimized and non-victimized wives. Violence and Victims, 7, 287-296.

Bell, M., Goodman, L. y Dutton, M. (2007). The dynamics of staying and leaving: Implications for battered women's emotional well-being and experiences of violence at the end of a year. Journal of Family Violence, 22, 413-428.

Ben-Porat, A. y Itzhaky, H. (2008). Factors that influence life satisfaction among battered women in shelters: Those who stay versus those who leave. Journal of Family Violence, 23, 597-604.

Brockner, J. y Rubin, J.Z. (1985). Entrapment in escalating conflicts: A social Psychological analysis. New York: Springer-Verlag.

Brown, J. (1997). Working toward freedom from violence. Journal of Violence Against Women, 3, 5-26.

Cavanaugh, M.M. y Gelles, R.J. (2005). The utility of male domestic violence offender typologies: New directions for research, policy and practice. Journal of Interpersonal Violence, 20, 155-166.

Choice, P. y Lamke, L.K. (1997). A conceptual approach to understanding abused women's stay/leave decisions. Journal of Family Issues, 18, 290-314.

Dutton, D.G. y Painter, S.L (1981). Traumatic bonding: The development of emotional attachment in battered women and other relationships of intermittent abuse. Victimology: An International Journal, 6, 139-155.

Dutton, D.G. y Painter, S.L. (1993). The battered woman syndrome: Effects of severity and intermittency of abuse. American Journal of Orthopsychiatry, 64, 614-622.

Echeburúa, E., Amor, P.J. y Corral, P., (2002). Mujeres maltratadas en convivencia prolongada con el agresor: variables relevantes. Acción Psicológica, 1, 135-150.

Fishbein, M. y Ajzen, I. (1975). Belief, attitude, intention, and behavior: An introduction to theory and research. Boston: Addison-Wesley.

Foa, E., Cascardi, M., Zoellner, L. y Feeny, N. (2000). Psychological and environmental factors associated with partner violence. Trauma, Violence, \& Abuse, 1, 67-91.

Gharaibeh, M. y Oweis, A. (2009). Why do Jordanian women stay in an abusive relationship. Implications for health and social well-being. Journal of Nursing Scholarship, 41, 376-384.

Goodman, L., Dutton, M., Weinfurt, K. y Cook, S. (2003). The Intimate Partner Violence Strategies Index: Development and application. Violence Against Women, 9, 163-186. 
Gondolf, E. (1988). The effect of batterer counseling on shelter outcome. Journal of Interpersonal Violence, 3 , 275-289.

Gordon, K.C., Burton, S. y Porter, L. (2004). Predicting the intentions of women in domestic violence shelters to return to partners. Does forgiveness play a role? Journal of Family Psychology, 18, 331-338.

Holtzworth-Munroe, A., Smutzler, N., Bates, L. y Sandin, E. (1997). Husband violence: Basic facts and clinical implications. Clinical handbook of marriage and couples interventions (pp. 129-156). Hoboken, NJ US: John Wiley \& Sons Inc.

Lesser, B. (1990). Attachment and situational factors influencing battered women's return to their mates following a shelter program. Research explorations in adult attachment (pp. 81-128). New York, NY US: Peter Lang Publishing.

Long, G.M. y McNamara, J.R. (1989). Paradoxical punishment as it related to the battered women syndrome. Behavior modification, 13, 192-205.

Pfouts, J.H. (1978). Violent families: coping responses of abused wives. Child Welfare, 57, 101-111.

Prochaska, J. O. y DiClemente, C. C. (1982). Transtheoretical therapy: Toward a more integrative model of change. Psychotherapy: Theory, Research and Practice, 19, 276-288.

Rhatigan, D.L. y Axsom, D.K. (2006). Using the investment model to understand battered women' commitment to abusive relationships. Journal of Family Violence, 21(2), 153-162.

Rhatigan, D.L., Street, A.E. y Axsom, D.K. (2006). A critical review of theories to explain violent relationship termination: Implications for research and intervention. Clinical Psychology Review, 26, 321-345.

Rhodes, N.R. y Baranoff, E.C. (1998). Why do battered women stay?: three decades of research. Aggression and Violent Behavior, 4, 391-406.

Rusbult, C. (1983). A longitudinal test of the investment model: The development (and deterioration) of satisfaction and commitment in heterosexual involvements. Journal of Personality and Social Psychology, 16, 172-186.

Rusbult, C.E. (1980). Commitment and satisfaction in romantic associations: A test of the investment model. Journal of Experimental and Social Psychology, 16, 172-186.

Salber, P.R. y Taliaferro, E. (2000). Reconocimiento y prevención de la violencia doméstica en el ámbito sanitario. Barcelona: Cedecs.

Seligman, M.E.P. (1975). Helplessness: On depression, development and death. San Francisco, CA: Freeman.

Stroshine, M. y Robinson, A. (2003). The decision to end abusive relationships: The role of offender characteristics. Criminal Justice and Behavior, 30, 97-117.

Strube, M.J. (1988). The Decision to Leave an Abusive Relationship: Empirical Evidence and Theoretical Issues. Psychological Bulletin, 2, 236-250.

Walker, L.E. (1979). The battered woman. New York: Harper and Row.

Walker, L.E. (1984). The battered woman syndrome. New York: Springer.

Manuscrito recibido: 21/05/2010

Revisión recibida: 08/06/2010

Manuscrito aceptado: 10/06/2010 